\title{
miR-148 family members are putative biomarkers for sepsis
}

\author{
LEI DONG $^{1 *}$, HONGWEI LI ${ }^{1 *}$, SHUNLI ZHANG ${ }^{2}$ and GUANZHENG YANG ${ }^{3}$ \\ ${ }^{1}$ Intensive Care Unit; Departments of ${ }^{2}$ Pediatrics and ${ }^{3}$ Respiratory Medicine, Jining No. 1 People's Hospital, \\ Jining, Shandong 272011, P.R. China
}

Received February 6, 2018; Accepted October 1, 2018

DOI: $10.3892 / \mathrm{mmr} .2019 .10174$

\begin{abstract}
Sepsis is a type of systemic inflammatory response caused by infection. The present study aimed to identify novel targets for the treatment of sepsis. We conducted bioinformatic analysis of the microarray Gene Expression Omnibus dataset GSE12624, which includes data on 34 patients with sepsis and 36 healthy individuals without sepsis. Differentially expressed genes (DEGs) in sepsis patients were identified using Bayesian methods included in the limma package in R. Correlations among the expression values of DEGs were analyzed using the weighted gene co-expression network analysis (WGCNA) to construct a co-expression network. Subsequently, the generated co-expression network was visualized using Cytoscape 3.3 software. Additionally, a protein-protein interaction (PPI) network was constructed based on all the DEGs using STRING. Finally, the integrated regulatory network was constructed based on DEGs, microRNAs (miRNAs) and transcription factors (TFs). A total of 407 DEGs were identified in the sepsis samples, including 227 upregulated DEGs and 180 downregulated DEGs. WGCNA grouped the DEGs into 13 co-expressed modules. Additionally, MAP3K8 and RPS6KA5 in the MEyellow module were enriched in the MAPK and TNF signaling pathways. In addition, the PPI network comprised 48 nodes and 112 edges, which included the pairs MAP3K8-RPS6KA5, MAP3K8-IL10, RPS6KA5-EXOSC4 and EXOSC4-EXOSC5. Lastly, the TF-miRNA-target DEG regulatory network was constructed based on eight TFs (NF- $\mathrm{B}$ ), seven miRNAs (miR152, miR-148A/B), and 52 TF-miRNA-target gene triplets (17 upregulated genes, including $M A P 3 K 8$, and 10 downregulated genes, including RPS6KA5). Our analysis showed that the members of the
\end{abstract}

Correspondence to: Ms. Guanzheng Yang, Department of Respiratory Medicine, Jining No. 1 People's Hospital, 6 Jiankang Road, Jining, Shandong 272011, P.R. China

E-mail: yanggz78@126.com

*Contributed equally

Key words: sepsis, differentially expressed genes, transcription factors, microRNAs, MAPK signaling pathway, TNF signaling pathway
miR-148 family (miR-148A/B and miR-152) are candidate biomarkers for sepsis.

\section{Introduction}

Sepsis is a type of systemic inflammatory response syndrome (SIRS) and is mediated by an immune response triggered by infection, which can progress from sepsis to severe sepsis and septic shock (1). Sepsis can lead to symptoms, including fever, increased heart rate, breathing rate and confusion (2). In 2015, the incidence rates of sepsis and severe sepsis in high-income countries were 0.44 and $0.27 \%$, respectively (3). Meanwhile, the mortality rates for sepsis were reported to be 3 and 75 cases per 1,000 individuals in two Chinese military hospitals (4). Sepsis remains difficult to predict, diagnose and treat (5). Thus, there is an urgent need to identify target genes and microRNAs (miRNAs) that can seve as biomarkers for sepsis.

Previous studies have demonstrated that proinflammatory cytokines, including interleukin-6 (IL-6) and tumor necrosis factor- $\alpha$ (TNF- $\alpha)$, are key mediators of inflammation during sepsis (6). A previous study showed that IL-6, IL-1 $\beta$, IL-8 and TNF- $\alpha$ levels were significantly upregulated in culture-proven sepsis groups relative to those in the control groups (7). Multiple studies have reported that target genes and miRNAs are involved in sepsis. For example, Nrf2 is a basic leucine zipper transcription factor (TF) that mediates the response to lipopolysaccharides (LPS) and TNF- $\alpha$ by activating NF- $\kappa \mathrm{B}$ production during experimental sepsis (8). In addition, procalcitonin (PCT) is elevated in patients with SIRS, and has been approved by the Food and Drug Administration (U.S. FDA) for the assessment of risk for developing severe sepsis in patients (9). Although PCT is closely associated with inflammation, there are some limitations specific for infection resulting in questionable efficacy as PCT can also be increased in noninfectious disease conditions (10). Generally, the concentration value of PCT $<0.5 \mathrm{ng} / \mathrm{ml}$ indicates a low risk while values of $0.5-2.0 \mathrm{ng} / \mathrm{ml}$ represent an intermediate likelihood of sepsis and/or septic shock. Wacker et al reported (11) that PCT had a modest diagnostic performance with $77 \%$ sensitivity and $79 \%$ specificity. Therefore, PCT is not specific for diagnosis in patients with values in the intermediate range. Importantly, multiple miRNAs have various biological functions in inflammation, metabolism and tumor progression. These candidate miRNAs show high accuracy and sensitivity, and are expected to be ideal biomarkers for sepsis. Evidence suggests that the 
sensitivity and specificity of miR-223 for predicting the occurrence of sepsis after urinary operation were higher than those of PCT $(12,13)$. In addition, miR-155 has been suggested to directly target key genes that are involved in LPS signaling, such as Fas-associated death domain protein, IкB kinase $\varepsilon$, and the receptor (TNFR superfamily)-interacting serine-threonine kinase 1 to enhance TNF- $\alpha$ production (14). Nevertheless, miR-125b targets the 3'-untranslated region of the TNF- $\alpha$ transcript (14). However, the fundamental mechanisms underlying the pathogenesis of sepsis remain unclear. Multiple mechanisms involving complex systemic inflammation networks, genetic polymorphisms, immune dysfunction, abnormal coagulant function, and host response to pathogenic microorganisms and their toxins are likely to be involved in sepsis. Therefore, the pathogenesis of sepsis warrants further investigation.

In the present study, we performed bioinformatics analysis to identify the differentially expressed genes (DEGs) in sepsis, as well as the TFs and miRNAs of these DEGs. Subsequently, an integrated regulatory network was constructed based on the DEGs, miRNAs and TFs. Finally, we investigated the interactions among the DEGs and TFs/miRNAs and their corresponding functions. Our current findings provided insights into the pathogenesis of sepsis and identified novel targets for the treatment of sepsis.

\section{Materials and methods}

Microarray data. The GSE12624 dataset was downloaded from the GEO database (http://www.ncbi.nlm.nih.gov/geo/) and contains gene expression data of 34 sepsis patients and 36 healthy individuals without sepsis. The inclusion criteria for the study are described in (15). The microarray platform was GPL4204 GE Healthcare/Amersham Biosciences CodeLink UniSet Human I Bioarray. Raw data were available in TXT format.

Data preprocessing and identification of DEGs. The probes corresponded to gene symbols according to the latest annotation file from the NCBI gene database. When more than one probe corresponded to the same gene symbol, the expression level of the gene was calculated as the median of the two expression values. Subsequently, the data were fitted to a log-normal distribution using the $\log 2$ function, normalized using the median function, and compared with septic samples and non-septic samples using Bayesian methods from the limma package in $\mathrm{R}$ (Linear Models for Microarray Data, http://www.bioconductor. org/packages/release/bioc/html/limma.html). Finally, llog fold change (FC) $>0.585$ and adjusted P-value $<0.05$ were used as the threshold values for considering the DEGs.

Identification of sepsis-related genes and modules based on WGCNA. WGCNA is a systematic method for identifying putative target genes involved in a disease. It is used to describe the correlation among genes by finding significant modules from high-throughput sequencing data (16). In the present study, WGCNA was performed based on the following analysis workflow. i) The correlations among the expression values of DEGs in the dataset were determined. A higher correlation value indicates higher consistency of gene expression in each dataset, which is a prerequisite for the construction of a WGCNA network. ii) The correlation matrix of gene co-expression values was constructed based on $S_{m n}=\left|\operatorname{cor}_{(m, n)}\right|$, where $S_{m n}$ indicates the correlation coefficient of co-expression patterns between genes $m$ and $n$. iii) The adjacency is defined as $\mathrm{a}_{\mathrm{mn}}=\operatorname{power}_{(\mathrm{Smn}, \beta)}$, which measures the pairwise correlation between the expression levels of two genes. iv) Adjacency functions for both weighted and unweighted networks require the user to choose threshold parameters. The threshold of $\geq 0.9$ was considered for the correlation coefficient between $\log _{2} \mathrm{k}$ (node count) and $\log _{2} \mathrm{p}(\mathrm{k}$ ) (frequency of node). v) The correlation matrix $S_{m n}$ was transformed to the adjacency matrix $a_{m n}$. Afterwards, the adjacency matrix $a_{m n}$ was transformed to a topological matrix using the following equation:

$$
\frac{\mathrm{l}_{\mathrm{mn}}+\mathrm{a}_{\mathrm{mn}}}{\min \left\{\mathrm{k}_{\mathrm{m}}, \mathrm{k}_{\mathrm{n}}\right\}+1-\mathrm{a}_{\mathrm{mn}}}
$$

where $1_{\mathrm{mn}}$ indicates the sum of adjacency coefficient of the common edge between genes $m$ and $n$ and $k_{m}$ indicates sum of connection strengths of $m$ with the other network genes. vi) Gene significance (GS) measures were used to incorporate external information into the co-expression network. Module significance was determined by calculating the average |GS| for all genes in a module.

Gene Ontology (GO) enrichment and Kyoto Encyclopedia of Genes and Genomes (KEGG) pathway analyses of the key modules. The Database for Annotation, Visualization and Integration Discovery (DAVID, a public high-throughput functional annotation tool (version 6.8 , https://david-d.ncifcrf.gov/) is an online bioinformatics tool that can be used for functional annotation and microarray analysis by integrating data mining environments and analyzing gene lists (17). DEGs in the modules were used as input for DAVID, and GO and KEGG enrichment analyses were conducted using MEblue and MEyellow DEGs. P-value $<0.05$ and the enriched gene count $\geq 2$ were considered significant.

Construction of the PPI network and module analysis. The PPI network was constructed based on all the DEGs using STRING from a well-known online server (version 10.0, http://www.string-db.org/) (18). A combined score of $>0.4$ was defined as the threshold value for constructing the PPI network. The PPI network was visualized using Cytoscape software (version 3.2.0, http://cytoscape.org/) (19). In addition, MCODE (version 1.4.2, http://apps.cytoscape.org/apps/MCODE) in Cytoscape software was used to analyze the most significant module, with the threshold value of 5 (20).

Construction of the TF-miRNA-target DEGs regulatory network. The miRNA-target DEGs and TF-target DEGs were predicted using Overrepresentation Enrichment Analysis enrichment method in WebGestal (http://www.webgestalt.org/). Gene pairs with P-value $<0.05$ were integrated into the TF-miRNA-target DEGs regulatory network, which was visualized using Cytoscape.

\section{Results}

Sepsis-related genes and modules. A total of 407 DEGs, including 227 upregulated DEGs and 180 downregulated 

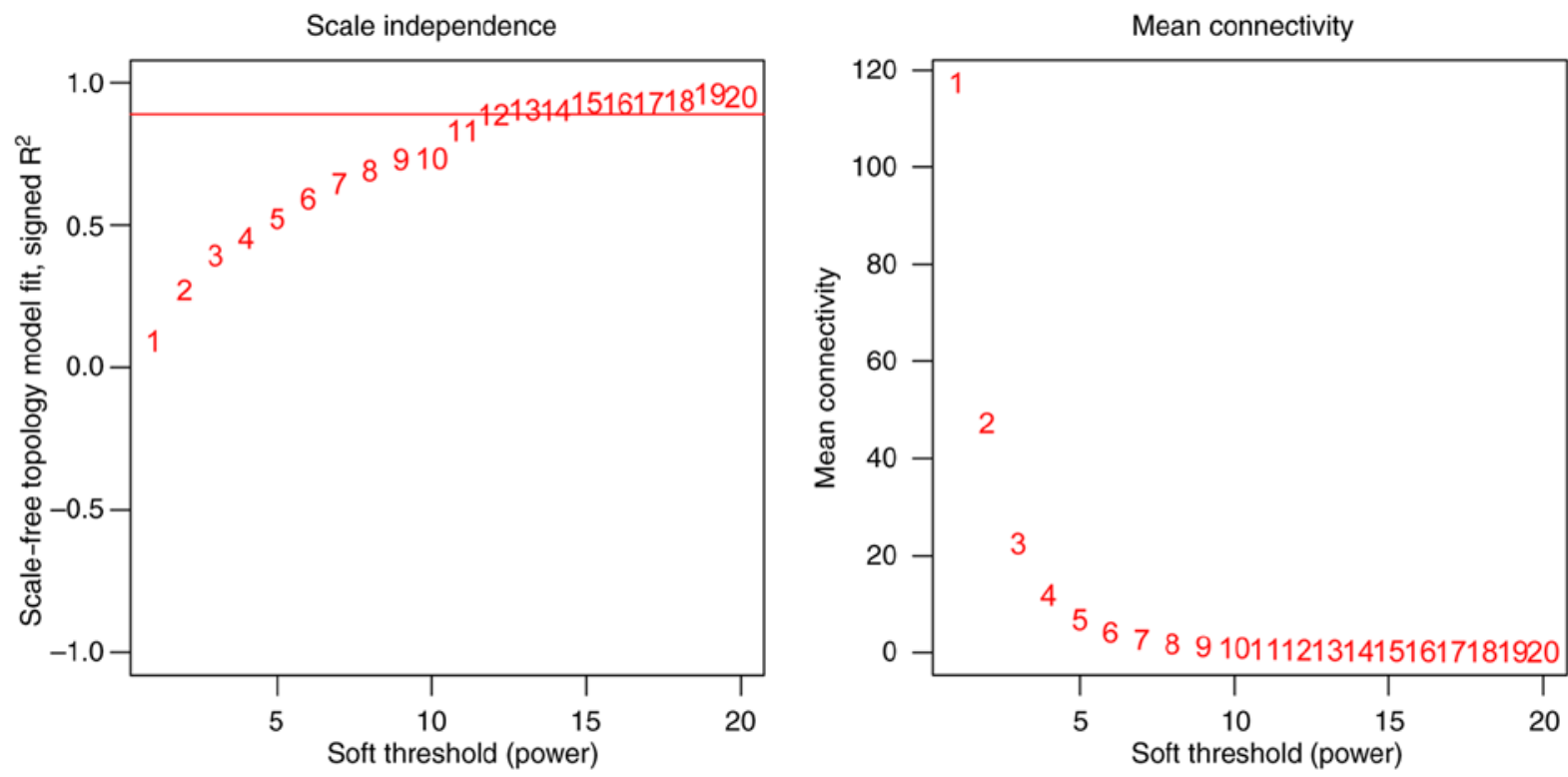

Figure 1. Power values (weighted threshold parameters) of the adjacency matrix. $x$-axis indicates the power value; $y$-axis indicates the square of the correlation coefficient between $\log _{2} \mathrm{k}$ (node count) and $\log _{2} \mathrm{p}(\mathrm{k})$ (frequency of node). The network approaches a scale-free network model as the square of correlation coefficient is increased. The red line indicates a square of correlation coefficient of 0.9 .

DEGs, were identified. According to the standard scale-free network model, the power value was set to 12 when the square of correlation coefficient was 0.9 (Fig. 1). The network conformed to a scale-free model when the square of the correlation coefficient square was set to the highest value. Subsequently, the WGCNA network was constructed under power $=12$. Gene cluster dendrogram was obtained according to dissTOM using the hierarchical clustering method. The dynamic tree cut method was employed to estimate the number of clusters in the dataset. Finally, the DEGs were divided into 13 co-expressed modules (Fig. 2), and genes in the grey module contained genes that could not clustered under the other modules. Subsequently, the most highly connected intramodular hub gene in each module was considered as the module representative. The analysis identified a total of 7 modules with correlation coefficients $>0.5$. The correlation coefficients of the MEblue and MEyellow modules were higher than 0.6. To ensure the reliability of the key network module, the IGSI was used to further identify two key modules (Fig. 3). Finally, the MEblue (Fig. 3A) and MEyellow (Fig. 3B) modules were defined as the key modules. All of DEGs in the two modules are shown in the Table I.

GO function and KEGG pathway analysis. A total of 66 DEGs were identified in the MEblue and MEyellow modules, including 46 upregulated DEGs and 20 downregulated DEGs. F8, PLAU and SERPING1 in the MEblue module were enriched with complement and coagulation cascades. EXOSC4 and EXOSC5 in the MEblue module were enriched in the RNA degradation pathway. MAP3K8 and RPS6KA5 in the MEyellow module were enriched in the MAPK and TNF signaling pathways. The GO functions of the genes in the two modules are shown in Table II.

PPI network based on the MEblue and MEyellow modules. The PPI network (Fig. 4) contained 48 nodes (genes)

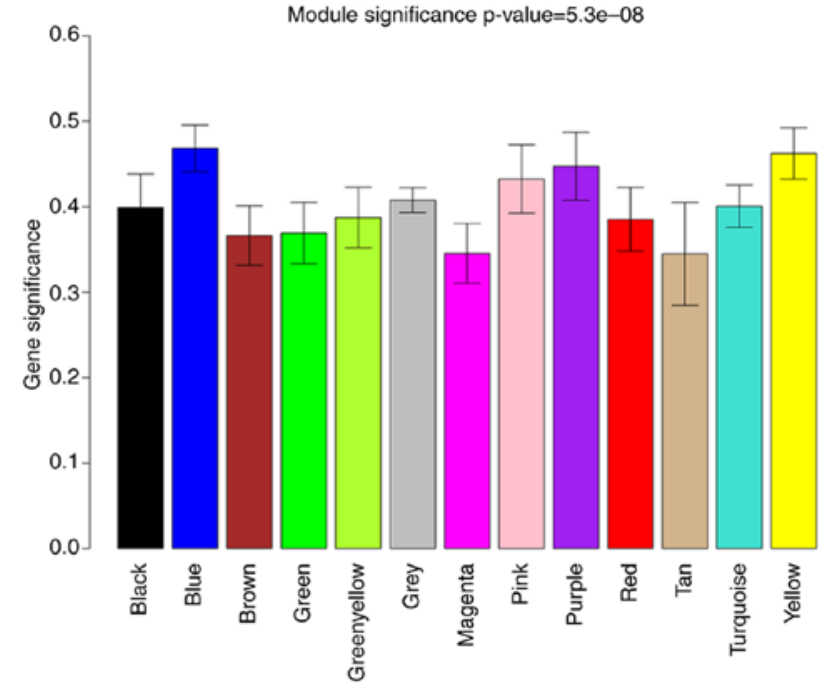

Figure 2. The 13 sepsis-related co-expressed modules after weighted gene co-expression network analysis. The horizontal axis represents each different color module; the vertical axis represents the correlation coefficient between genes in each module and disease status.

and 112 edges (protein-protein interrelations), such as MAP3K8-RPS6KA5, MAP3K8-IL10, RPS6KA5-EXOSC4 and EXOSC4-EXOSC5). One sub-network (hub module) had an MCODE score $\geq 5$ and comprised 7 nodes (e.g. IL10) and 15 edges (Fig. 4).

miRNA-TF-target gene regulatory network. Overall, the analysis identified $8 \mathrm{TFs}(\mathrm{NF}-\kappa \mathrm{B})$ and 7 miRNAs (miR152 and miR-148A/B), which comprised 52 TF-miRNA-target gene pairs (17 upregulated genes, such as $M A P 3 K 8$ and 10 downregulated genes, such as RPS6KA5) and were used to construct an miRNA-TF-target gene regulatory network (Fig. 5). 

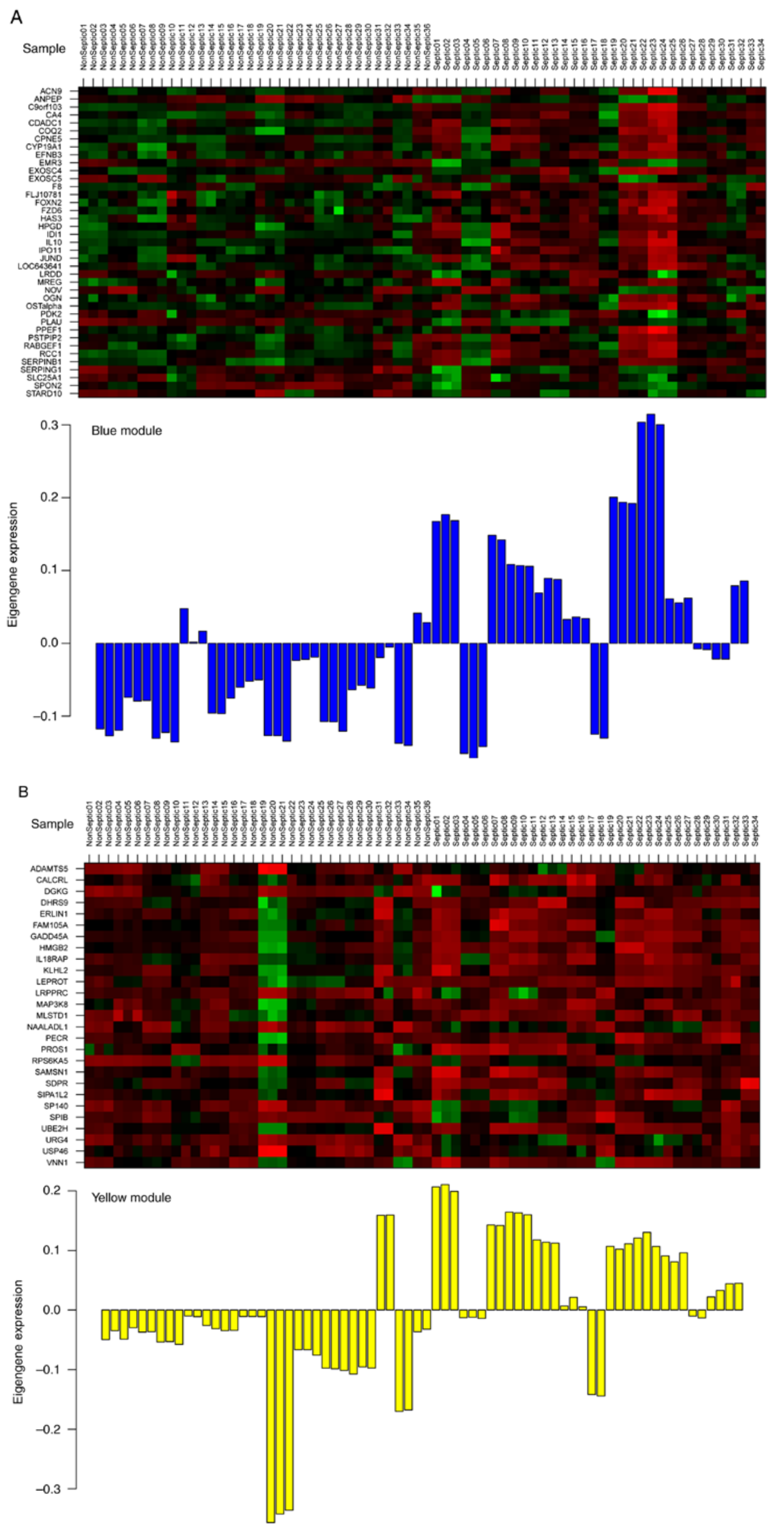

Figure 3. The two key modules [(A) MEblue and (B) MEyellow] were identified by calculating IGene significance (GS)| values. 
Table I. Differentially expressed genes in the MEblue and MEyellow modules.

\begin{tabular}{|c|c|c|c|c|c|}
\hline Genes & Module & Description & Genes & Module & Description \\
\hline ACN9 & blue & up & ADAMTS5 & yellow & down \\
\hline ANPEP & blue & down & $C A L C R L$ & yellow & up \\
\hline$C A 4$ & blue & up & $D G K G$ & yellow & down \\
\hline$C D A D C 1$ & blue & up & DHRS9 & yellow & up \\
\hline$C O Q 2$ & blue & up & ERLIN1 & yellow & up \\
\hline CPNE5 & blue & up & FAM105A & yellow & up \\
\hline СYP19A1 & blue & up & $F A R 2$ & yellow & up \\
\hline EFNB3 & blue & up & $G A D D 45 A$ & yellow & up \\
\hline EMR3 & blue & down & $H M G B 2$ & yellow & up \\
\hline EXOSC4 & blue & up & ILI8RAP & yellow & up \\
\hline EXOSC 5 & blue & down & KLHL2 & yellow & up \\
\hline$F 8$ & blue & up & LEPROT & yellow & up \\
\hline FOXN2 & blue & up & $L R P P R C$ & yellow & down \\
\hline FZD6 & blue & up & $M A P 3 K 8$ & yellow & up \\
\hline HAS3 & blue & up & $N A A L A D L 1$ & yellow & down \\
\hline$H P G D$ & blue & up & $P E C R$ & yellow & up \\
\hline IDII & blue & up & PROS1 & yellow & up \\
\hline$I D N K$ & blue & up & RPS6KA5 & yellow & down \\
\hline ILIO & blue & up & SAMSN1 & yellow & up \\
\hline IPOII & blue & up & $S D P R$ & yellow & up \\
\hline JUND & blue & up & SIPAIL2 & yellow & up \\
\hline LOC643641 & blue & up & SP140 & yellow & down \\
\hline$M R E G$ & blue & up & $S P I B$ & yellow & down \\
\hline$N O V$ & blue & down & $U B E 2 H$ & yellow & up \\
\hline$O G N$ & blue & up & $U R G C P$ & yellow & down \\
\hline$P D K 2$ & blue & down & USP46 & yellow & down \\
\hline$P I D D$ & blue & down & VNN1 & yellow & up \\
\hline$P L A U$ & blue & down & & & \\
\hline PNMAL1 & blue & up & & & \\
\hline PPEF1 & blue & up & & & \\
\hline PSTPIP2 & blue & up & & & \\
\hline$R A B G E F 1$ & blue & up & & & \\
\hline$R C C 1$ & blue & up & & & \\
\hline SERPINBI & blue & up & & & \\
\hline SERPING1 & blue & down & & & \\
\hline$S L C 25 A 1$ & blue & down & & & \\
\hline SLC51A & blue & up & & & \\
\hline SPON2 & blue & down & & & \\
\hline STARD10 & blue & down & & & \\
\hline
\end{tabular}

Up, upregulated; down, downregulated.

\section{Discussion}

In the present study, we identified a total of 407 DEGs in the sepsis samples, including 227 upregulated DEGs and 180 downregulated DEGs. Subsequently, these DEGs were grouped into 13 co-expressed modules after WGCNA. Additionally, MEblue and MEyellow modules with a correlation coefficient $>0.6$ were defined as the key modules; these modules included 6 upregulated and 20 downregulated DEGs. EXOSC4 and EXOSC5 in the
MEblue module were enriched in the RNA degradation pathway. $M A P 3 K 8$ and RPS6KA5 in the MEyellow module were enriched in the MAPK and TNF signaling pathways. In addition, the resulting PPI network comprised 48 nodes and 112 edges such as MAP3K8-RPS6KA5, MAP3K8-IL10, RPS6KA5-EXOSC4 and EXOSC4-EXOSC5. Finally, the analysis identified 8 TFs (NF- $\mathrm{B})$ and 7 miRNAs (miR-152 and miR-148A/B) that corresponded to 52 TF-miRNA-target gene pairs (17 upregulated genes, such as $M A P 3 K 8$ and 10 downregulated genes, such as RPS6KA5). 


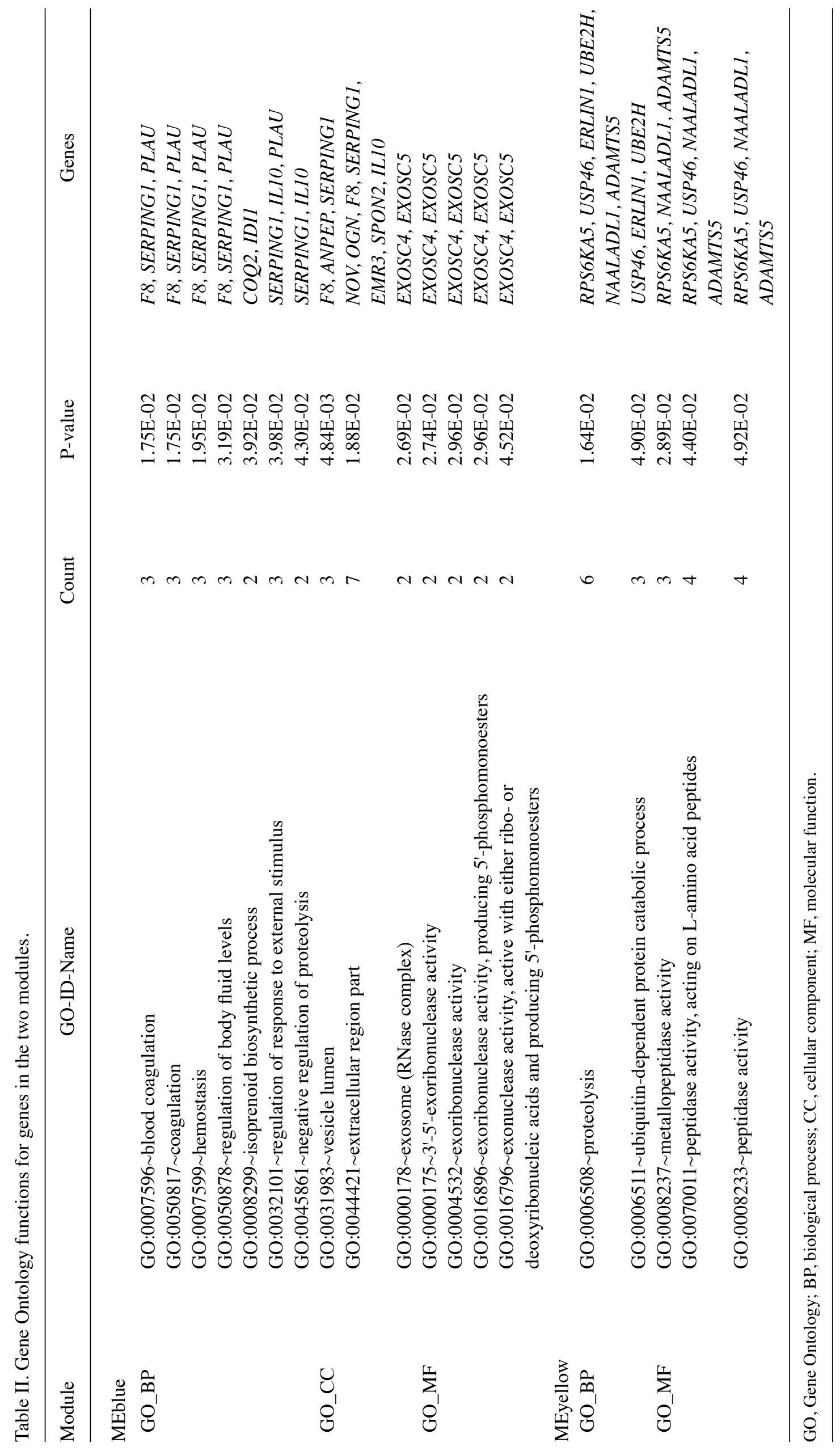




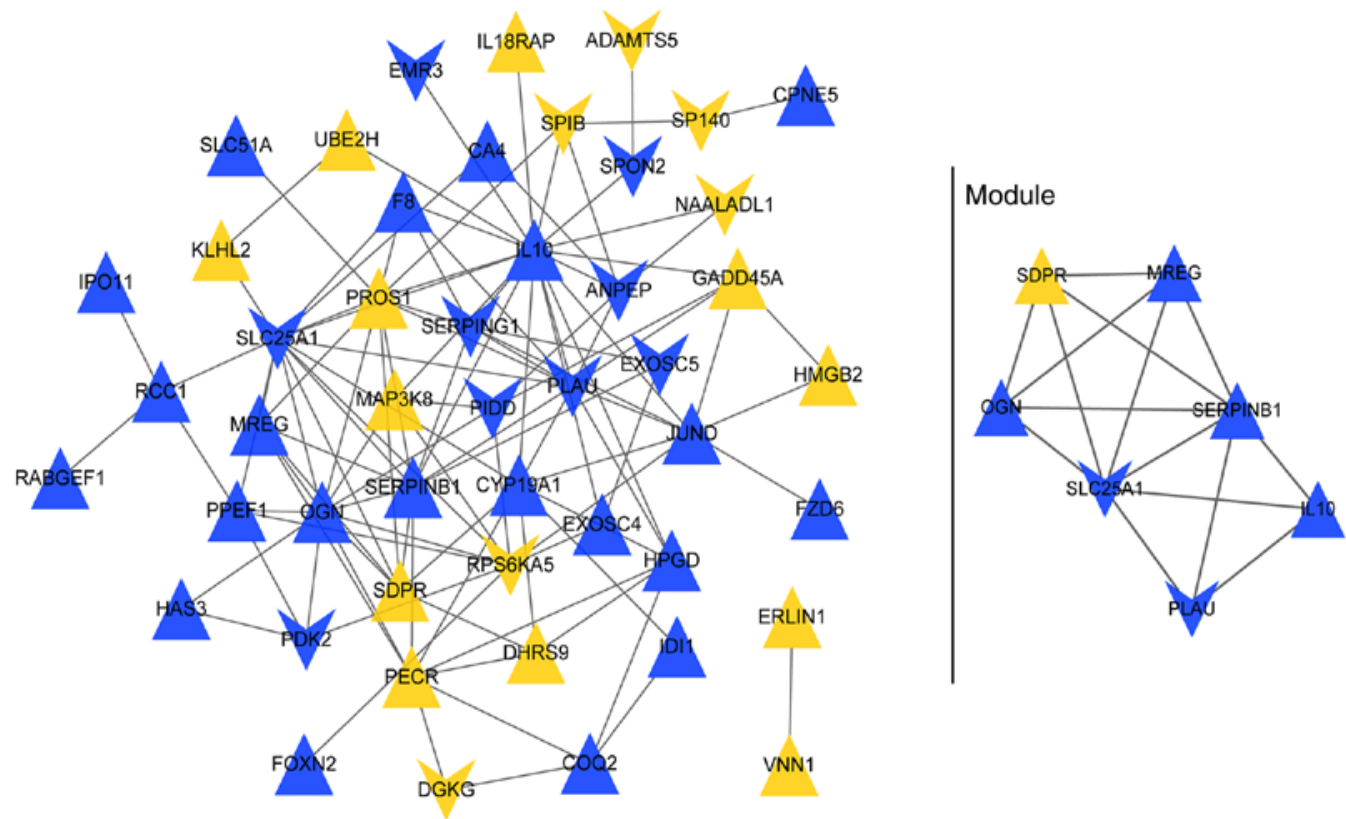

Figure 4. The protein-protein interaction (PPI) network based on the differentially expressed genes (DEGs) in the MEblue and MEyellow modules. Triangles indicate upregulated DEGs, and arrows indicate downregulated DEGs. Yellow and blue colors indicate genes in the MEyellow and MEblue modules, respectively.

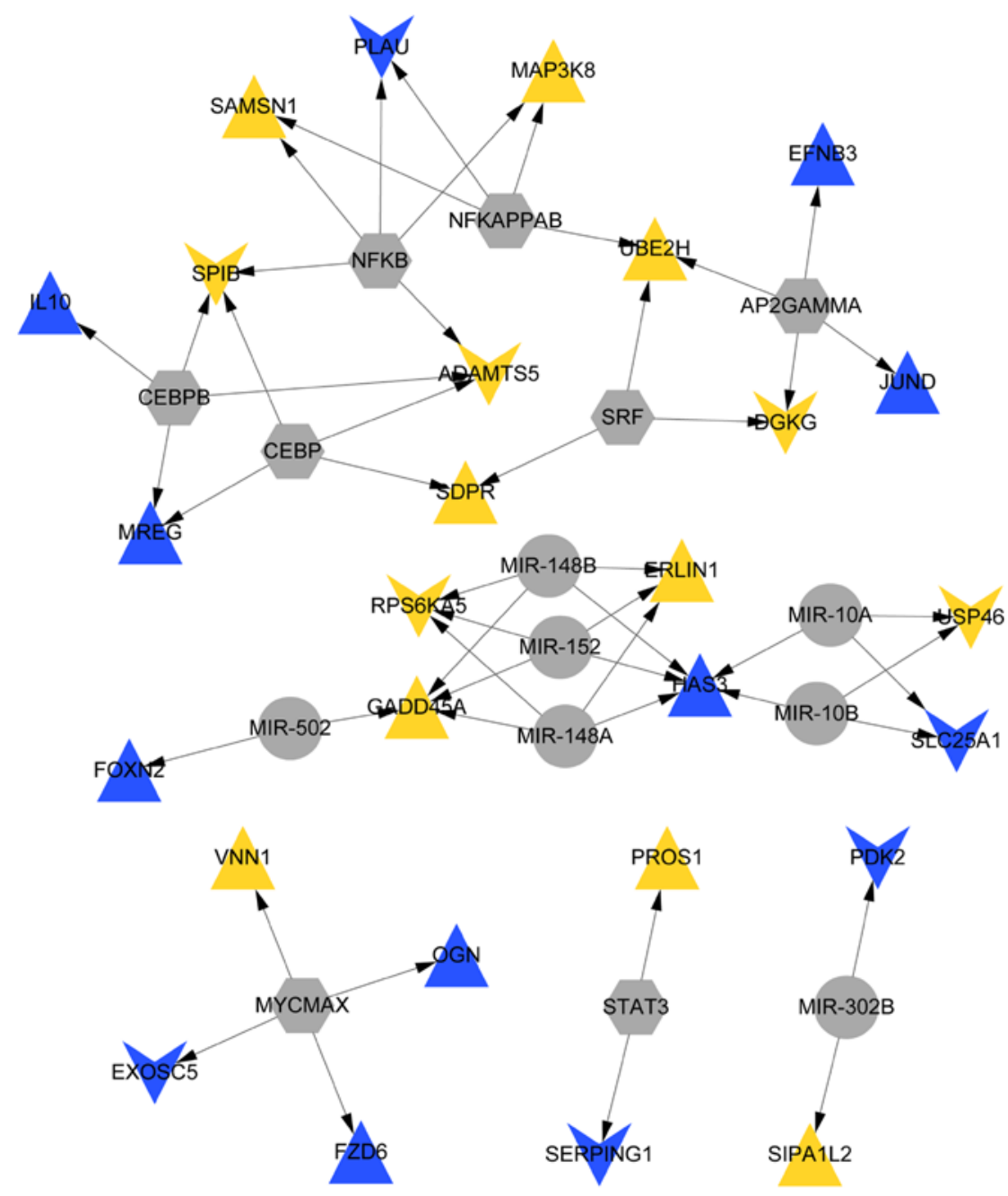

Figure 5. Constructed TF-miRNA-target DEG regulatory network. Triangles indicate upregulated DEGs; arrows indicate downregulated DEGs; hexagons indicate TFs; and the circles indicate miRNAs. Yellow and blue colors indicate genes in the MEyellow and MEblue modules, respectively. Arrows indicate the direction of regulation. TF, transcription factor; DEG, differentially expressed gene. 
$M A P 3 K 8$ is a serine-threonine kinase that plays a critical role in innate immunity and is known to induce tumor necrosis factor (TNF) production by activating ERK (21). In addition, TNF- $\alpha$ has been implicated as a key mediator in inflammation, morbidity and mortality associated with sepsis. TNF- $\alpha$ has been demonstrated to be responsible for the initial hypothermia and lethality in septic mice. (22). In addition, host reactions during sepsis, septic shock, and multiple organ failure are associated with increased TNF production in humans (23). TNF- $\alpha$ is a strong pro-inflammatory cytokine associated with septic patients and has been considered as a target for the treatment of sepsis (24). In the present study, $M A P 3 K 8$ expression levels were found to be upregulated in sepsis samples relative to those of the control samples. As indicated above, $M A P 3 K 8$ induces TNF production, consistent with increased TNF levels in the sepsis samples in the present study. Importantly, MAP3K8 and RPS6KA5 in the MEyellow module were enriched in the MAPK and TNF signaling pathways. In addition, MAP3K8 interacts with IL10 based on the constructed PPI network. IL10 is an anti-inflammatory agent that can improve disease outcome in the model of sepsis syndrome (25). Therefore, these findings indicate that $M A P 3 K 8$ is involved in sepsis through the MAPK and TNF signaling pathways. The nuclear transcription factor $\mathrm{NF}-\kappa \mathrm{B}$ is known to be activated following hemorrhagic shock and sepsis (26). In the present study, NF- $\kappa \mathrm{B}$ acts as the upstream TF of the MAP $3 K 8$ gene. Proinflammatory cytokines, such as TNF- $\alpha$ and IL-1, activate important signaling pathways. In particular, cytokines activate members of the NF- $\kappa \mathrm{B}$ group of TFs, which play central roles in inflammation and innate immunity (27). Activation of NF- $\kappa \mathrm{B}$ and other TFs involved in the innate immune/inflammatory response can upregulate the expression of various genes, such as MMP-9, VEGF and $T N F$ (28). Therefore, $M A P 3 K 8$ is potentially involved in sepsis through the activation of NF- $\kappa \mathrm{B}$ and is likely to be involved in the MAPK and TNF signaling pathways.

In the PPI network, RPS6KA5 interacted with MAP3K8, and these two genes were enriched in the MAPK and TNF signaling pathways. RPS6KA5, also known as mitogen- and stress-activated protein kinase $1(M S K 1)$, is a downstream target of both p38 and ERK1/2 (29). RPS6KA5 stimulates the transcription of various pro-inflammatory genes, such as IL-6, IL- 8 and TNF- $\alpha$, by activating TFs (30). Therefore, RPS6KA5 was associated with sepsis through the MAPK and TNF signaling pathways. In the miRNA-TF-target gene regulatory network, RPS6KA5 was the target gene of miR-152, miR-148A and miR-148B. A previous study indicated that members of the miR-148 family (miR-148A, miR-148B and miR-152) negatively regulated antigen presentation and Toll-like receptor (TLR)-triggered cytokine secretion in dendritic cells (31). TLRs are a class of proteins that play key roles in the innate immune system and secrete proinflammatory cytokines, such as TNF- $\alpha$, IL-6 and IL-12 (32,33). In addition, soluble TLR2 is a biomarker for sepsis in critically ill patients with multi-organ failure within $12 \mathrm{~h}$ of ICU admission (34). Although there was no direct evidence to identify that miR-148 is better than PCT or TLR2, miRNAs with high accuracy and sensitivity, are expected to be ideal biomarkers for sepsis (13). Thus, the receiver operating characteristic (ROC) curve of the miR-148 family (including sensitivity and specificity) should be compared with those of PCT or TLR2 in diagnostic performance of sepsis patients. It is one of the limitation of the present study. Therefore, members of the miR-148 family (miR-148A, miR-148B and miR-152) may be candidate biomarkers for sepsis.

The present study has certain limitations. First, limited samples were collected from the sepsis patients, and experimental validation of the results was not performed. PCR or western blotting will be performed in subsequent studies to verify the findings. In addition, experiments should be conducted to verify whether RPS6KA5 is a target of miR-148A/B and miR-152 in sepsis. In addition, the microarray dataset GSE12624 from the Gene Expression Omnibus only included 34 patients with sepsis and 36 healthy individuals without sepsis. Therefore, correlation among the miR-148 family (miR-148A/B and miR-152), and the type of infection was not performed. In addition, an ROC curve of the miR-148 family should be assayed in the diagnostic performance of sepsis patients. However, the present results will not be affected by these limitations.

Therefore, MAP $3 K 8$ is potentially induced during sepsis through $N F-\kappa B$ activation and is potentially involved in the MAPK and TNF signaling pathways. Meanwhile, RPS6KA5 interacted with MAP3K8 in the PPI network and was also found to be enriched in the MAPK and TNF signaling pathways. Members of the miR-148 family (miR-148A/B and miR-152) are candidate biomarkers for sepsis.

\section{Acknowledgements}

Not applicable.

\section{Funding}

No funding was received.

\section{Availability of data and materials}

All data generated or analyzed during this study are included in the published article.

\section{Authors' contributions}

Conception and design of the research were carried out by LD, HL and GY. Data collection, analysis and interpretation were conducted by SZ. Drafting of the manuscript was performed by LD and HL. Revision of the manuscript for important intellectual content was conducted by GY. All authors read and approved the manuscript and agree to be accountable for all aspects of the research in ensuring that the accuracy or integrity of any part of the work are appropriately investigated and resolved.

\section{Ethics approval and consent to participate}

Not applicable.

\section{Patient consent for publication}

Not applicable. 


\section{Competing interests}

The authors declare that they have no competing interests.

\section{References}

1. Rhodes A, Evans LE, Alhazzani W, Levy MM, Antonelli M, Ferrer R, Kumar A, Sevransky JE, Sprung CL, Nunnally ME, et al: Surviving sepsis campaign: International guidelines for management of sepsis and septic shock: 2016. Crit Care Med 45: 486-552, 2017.

2. Tsai D, Stewart P, Goud R, Gourley S, Hewagama S Krishnaswamy S, Wallis SC, Lipman J and Roberts JA: Total and unbound ceftriaxone pharmacokinetics in critically ill Australian Indigenous patients with severe sepsis. Int J Antimicrob Agents 48: 748-752, 2016.

3. Fleischmann C, Scherag A, Adhikari NK, Hartog CS, Tsaganos T, Schlattmann P, Angus DC and Reinhart K; International Forum of Acute Care Trialists: Assessment of global incidence and mortality of hospital-treated sepsis.current estimates and limitations. Am J Respir Crit Care Med 193: 259-272, 2016.

4. Cheng W, Wang S, Shen C, Zhao D, Li D and Shang Y: Epidemiology of hospitalized burns patients in china: A Systematic Review. Burn Open 2: 8-16, 2017.

5. Mitra P, Guha D, Nag SS, Mondal BC and Dasgupta S: Role of plasma fibrinogen in diagnosis and prediction of short term outcome in neonatal sepsis. Indian J Hematol Blood Transfus 33: 195-199, 2017

6. Kang S, Tanaka T, Masuda $\mathrm{K}$ and Kishimoto T: Implications of IL-6 Targeting Therapy for Sepsis. Immunotherapy (Los Angel) 3: 138, 2017.

7. Kurt AN, Aygun AD, Godekmerdan A, Kurt A, Dogan Y and Yilmaz E: Serum IL-1beta, IL-6, IL-8, and TNF-alpha levels in early diagnosis and management of neonatal sepsis. Mediators Inflamm 2007: 31397, 2007.

8. Thimmulappa RK, Lee H, Rangasamy T, Reddy SP, Yamamoto M, Kensler TW and Biswal S: Nrf2 is a critical regulator of the innate immune response and survival during experimental sepsis. J Clin Invest 116: 984-995, 2006

9. Schuetz P, Birkhahn R, Sherwin R, Jones AE, Singer A, Kline JA Runyon MS, Self WH, Courtney DM, Nowak RM, et al: Serial procalcitonin predicts mortality in severe sepsis patients: Results from the multicenter procalcitonin monitoring sepsis (MOSES) Study. Crit Care Med 45: 781-789, 2017.

10. Riedel S: Procalcitonin and the role of biomarkers in the diagnosis and management of sepsis. Diagn Microbiol Infect Dis 73: 221-227, 2012

11. Wacker C, Prkno A, Brunkhorst FM and Schlattmann P Procalcitonin as a diagnostic marker for sepsis: A systematic review and meta-analysis. Lancet Infect Dis 13: 426-435, 2013.

12. Wu X, Yang J, Yu L and Long D: Plasma miRNA-223 correlates with risk, inflammatory markers as well as prognosis in sepsis patients. Medicine (Baltimore) 97: e11352, 2018.

13. Bao YYX and Chen Z: The early diagnostic value of microRNA-223 for patients with complication of sepsis after ureteroscopic lithotrity. Chin J Integr Tradit West Med Intensive Crit Care 24: 465-468, 2017 (In Chinese).

14. Tili E, Michaille JJ, Cimino A, Costinean S, Dumitru CD, Adair B, Fabbri M, Alder H, Liu CG, Calin GA and Croce CM: Modulation of miR-155 and miR-125b levels following lipopolysaccharide/TNF-alpha stimulation and their possible roles in regulating the response to endotoxin shock. J Immunol 179: 5082-5089, 2007.

15. Menges T, König IR, Hossain H, Little S, Tchatalbachev S, Thierer F, Hackstein H, Franjkovic I, Colaris T, Martens F, et al: Sepsis syndrome and death in trauma patients are associated with variation in the gene encoding tumor necrosis factor. Crit Care Med 36: 1456-1462, e1-e6, 2008.

16. Langfelder $P$ and Horvath S: WGCNA: An R package for weighted correlation network analysis. BMC Bioinformatics 9 : $559,2008$.

17. Huang W, Sherman BT and Lempicki RA: Systematic and integrative analysis of large gene lists using DAVID bioinformatics resources. Nat Protoc 4: 44-57, 2009.
18. Szklarczyk D, Franceschini A, Wyder S, Forslund K, Heller D, Huerta-Cepas J, Simonovic M, Roth A, Santos A, Tsafou KP, et al: STRING v10: Protein-protein interaction networks, integrated over the tree of life. Nucleic Acids Res 43 (D1): D447-D452, 2015.

19. Shannon P, Markiel A, Ozier O, Baliga NS, Wang JT, Ramage D, Amin N, Schwikowski B and Ideker T: Cytoscape: A software environment for integrated models of biomolecular interaction networks. Genome Res 13: 2498-2504, 2003

20. Bandettini WP, Kellman P, Mancini C, Booker OJ, Vasu S, Leung SW, Wilson JR, Shanbhag SM, Chen MY and Arai AE: MultiContrast Delayed Enhancement (MCODE) improves detection of subendocardial myocardial infarction by late gadolinium enhancement cardiovascular magnetic resonance: A clinical validation study. J Cardiovasc Magn Reson 14: 83, 2012.

21. Mielke LA, Elkins KL, Wei L, Starr R, Tsichlis PN, O'Shea JJ and Watford WT: Tumor progression locus 2 (Map3k8) is critical for host defense against Listeria monocytogenes and IL-1 beta production. J Immunol 183: 7984-7993, 2009.

22. Leon LR, White AA and Kluger MJ: Role of IL-6 and TNF in thermoregulation and survival during sepsis in mice. Am J Physiol 275: R269-R277, 1998.

23. Stuber F, Udalova IA, Book M, Drutskaya LN, Kuprash DV, Turetskaya RL, Schade FU and Nedospasov SA: -308 tumor necrosis factor (TNF) polymorphism is not associated with survival in severe sepsis and is unrelated to lipopolysaccharide inducibility of the human TNF promoter. J Inflamm 46: 42-50, 1995-1996.

24. Riedemann NC, Guo RF and Ward PA: Novel strategies for the treatment of sepsis. Nat Med 9: 517-524, 2003.

25. OberholzerC,Oberholzer A,BahjatFR,Minter RM, TannahillCL, Abouhamze A, LaFace D, Hutchins B, Clare-Salzler MJ and Moldawer LL: Targeted adenovirus-induced expression of IL-10 decreases thymic apoptosis and improves survival in murine sepsis. Proc Natl Acad Sci USA 98: 11503-11508, 2001

26. Filgueiras LR Jr, Martins JO, Serezani CH, Capelozzi VL, Montes MBA and Jancar S: Sepsis-induced acute lung injury (ALI) is milder in diabetic rats and correlates with impaired NFkB activation. PLoS One 7: e44987, 2012.

27. Li Q and Verma IM: NF-kappaB regulation in the immune system. Nat Rev Immunol 2: 725-734, 2002.

28. Karin M: Nuclear factor-kappaB in cancer development and progression. Nature 441: 431-436, 2006

29. Jiang C, Yu L, Tu Q, Zhao Y, Zhang $\mathrm{H}$ and Zhao S: Assignment of a member of the ribosomal protein S6 kinase family, RPS6KA5, to human chromosome $14 \mathrm{q} 31-->\mathrm{q} 32.1$ by radiation hybrid mapping. Cytogenet Cell Genet 87: 261-262, 1999.

30. Funding AT, Johansen C, Kragballe K, Otkjaer K, Jensen UB, Madsen MW, Fjording MS, Finnemann J, Skak-Nielsen T, Paludan SR and Iversen L: Mitogen- and stress-activated protein kinase 1 is activated in lesional psoriatic epidermis and regulates the expression of pro-inflammatory cytokines. J Invest Dermatol 126: 1784-1791, 2006.

31. Liu X, Zhan Z, Xu L, Ma F, Li D, Guo Z, Li N and Cao X: MicroRNA-148/152 impair innate response and antigen presentation of TLR-triggered dendritic cells by targeting CaMKII $\alpha$. J Immunol 185: 7244-7251, 2010.

32. Aslam R, Speck ER, Kim M, Crow AR, Bang KW, Nestel FP, Ni H, Lazarus AH, Freedman J and Semple JW: Platelet Toll-like receptor expression modulates lipopolysaccharide-induced thrombocytopenia and tumor necrosis factor-alpha production in vivo. Blood 107: 637-641, 2006.

33. Eidson LN, Inoue K, Young LJ, Tansey MG and Murphy AZ Toll-like receptor 4 mediates morphine-induced neuroinflammation and tolerance via soluble tumor necrosis factor signaling. Neuropsychopharmacology 42: 661-670, 2017.

34. Holst B, Szakmany T, Raby AC, Hamlyn V, Durno K, Hall JE and Labéta MO: Soluble Toll-like receptor 2 is a biomarker for sepsis in critically ill patients with multi-organ failure within $12 \mathrm{~h}$ of ICU admission. Intensive Care Med Exp 5: 2, 2017.

This work is licensed under a Creative Commons Attribution-NonCommercial-NoDerivatives 4.0 International (CC BY-NC-ND 4.0) License. 